\title{
Análise do desgaste e da sobrecarga de cuidadores/ familiares de idosos com doença de Alzheimer causado pelos sintomas psicológicos e comportamentais*
}

\author{
Analysis of caregiver burden of elderly with \\ Alzheimer disease caused by behavioral \\ psychological symptons of dementia
}

\author{
Blenda Kimie Arakaki ${ }^{1}$, Jaqueline Namy de Souza Tsubaki ${ }^{1}$, Paulo \\ Caramelli ${ }^{2}$, Ricardo Nitrini ${ }^{3}$, Marcia Maria Pires Camargo Novelli ${ }^{4,5}$
}

ARAKAKI, B. K.; TSUBAKI, J. N. S.; CARAMELLI, P.; NITRINI, R.; NOVELLI, M. M. P. C. Análise do desgaste e da sobrecarga de cuidadores/familiares de idosos com doença de Alzheimer causado pelos sintomas psicológicos e comportamentais. Rev. Ter. Ocup. Univ. São Paulo, v. 23, n. 2, p. 113-121, maio/ago. 2012.

RESUMO: A demência da doença de Alzheimer (DA) se caracteriza como uma doença crônica e degenerativa que compromete o indivíduo em diversos domínios, dentre eles o comportamento sendo que essas alterações podem causar desgaste e sobrecarga no cuidador/familiar. Objetivou-se analisar o nível de sobrecarga e de desgaste do cuidador/familiar em função da presença e intensidade dos "sintomas psicológicos e comportamentais da demência" (SPCD). Como instrumentos de avaliação foram utilizados, o Inventário Neuropsiquiátrico (INP), com a escala de desgaste do cuidador (INP-D), Inventário de Sobrecarga do Cuidador e um questionário de caracterização sociodemográfica. Foram entrevistados 30 cuidadores de idosos com DA provável segundo os critérios do NINCDS-ADRDA, divididos em grupos de gravidade de acordo com os critérios do DSM-III-R, sendo 15 (grau leve) e 15 (grau moderado). Os sintomas mais prevalentes, na fase leve, foram ansiedade e depressão e na fase moderada, ansiedade e agitação. Os sintomas que mais se correlacionaram com o desgaste do cuidador, na fase leve, foram agitação, seguido de desinibição e na fase moderada, alucinação e apatia. Em relação a sobrecarga, a correlação encontrada foi com delírio, na fase leve, e apatia na fase moderada. Concluindo, verificou-se que os SPCD que mais sobrecarregam e desgastam os cuidadores diferenciam-se de acordo com a gravidade da doença e que o conhecimento deste impacto nos permite refletir sobre ações que visem a implementação de politicas publicas no atendimento e acompanhamento do idoso e de seus cuidadores/familiares.

DESCRITORES: Doença de Alzheimer; Cuidadores/utilização; Cuidadores/psicologia; Idoso; Família; Sintomas comportamentais/diagnóstico; Sintomas comportamentais/psicologia.

\footnotetext{
* Este trabalho é parte da Iniciação Cientifica com bolsa do CNPQ - 2008-2009. Trabalho apresentado no XVI Congresso de Iniciação Cientifica da UNIFESP, 2009.

1. Terapeuta Ocupacional graduada pela Universidade Federal de São Paulo.

2. Professor Adjunto da Escola de Medicina da Universidade Federal de Minas Gerais.

3. Professor Titular, Departamento de Neurologia da Faculdade de Medicina da Universidade de São Paulo.

4. Professor Adjunto no curso de Terapia Ocupacional da Universidade Federal de São Paulo - Campus Baixada Santista.

5. Líder do Núcleo Interprofissional de Pesquisa e Atendimento no Envelhecimento (NIPAE) da Universidade Federal de São Paulo - Campus Baixada Santista.

Endereço para correspondência: Marcia Maria Pires Camargo Novelli. Universidade Federal de São Paulo - Campus Baixada Santista. Departamento de Gestão e Cuidados em Saúde. Rua Silva Jardim, 136 - Vila Mathias - CEP 11.015-020. Santos - São Paulo. E-mail: mnovelli@uol.com.br
} 


\section{INTRODUÇÃO}

$\mathrm{C}$ om o aumento da expectativa de vida tornamse cada vez mais frequentes as doenças neurodegenerativas, como as síndromes demenciais (CAMARANO, 2006; FREITAS et al., 2008) .

A demência da doença de Alzheimer (DA) é a causa mais frequente de demência, responsável por mais de $50 \%$ dos casos, na faixa etária igual ou superior a 65 anos (HERRERA et al., 2002). O quadro clínico é caracterizado por alterações cognitivas e comportamentais, com preservação do funcionamento motor e sensorial até as fases mais avançadas da doença (CARAMELLI; BARBOSA, 2002; FREITAS et al., 2008; FROTA et al., 2011).

À medida que a DA progride, surge à demanda por cuidados especiais, função normalmente desempenhada pelos cuidadores. A família é apontada como o principal suporte quando o idoso adoece, devido à ausência de uma rede de suporte formal adequada (NOVELLI et al., 2010).

Os cuidadores informais irão assumir, dentro de suas capacidades, os cuidados relacionados ao idoso portador de síndrome demencial (GARRIDO; MENEZES, 2004; CRUZ; HAMDAN, 2008).

O desenvolvimento dos sintomas psicológicos e comportamentais é um dos problemas que emerge com frequência durante a evolução da doença. Assim, a vida do cuidador passa a ser influenciada pelos aspectos cognitivos, funcionais e comportamentais da demência (GARRIDO; MENEZES, 2004).

Sintomas psicológicos e comportamentais da demência (SPCD) referem-se ao conjunto de sintomas e sinais relacionados a transtornos da percepção, do conteúdo do pensamento, do humor ou do comportamento que ocorrem frequentemente em pessoas com demência de qualquer etiologia (CARAMELLI; BOTTINO, 2007). Os SPCD são também chamados de aspectos não cognitivos da demência.

As alterações psicológicas e comportamentais nas demências apresentam-se principalmente por apatia, isolamento social, sintomas depressivos, ansiedade, delírios, agitação/agressividade, alucinações, mania, irritabilidade, falsas interpretações, perambulação que também pode ser nomeada como comportamento motor aberrante, reações catastróficas, desinibição do comportamento e alterações nos ciclos de sono e vigília. A frequência desses sintomas em idosos com demência é bastante elevada (ANGUINAH et al., 2007).

Eles estão associados com maior estresse, desgaste e sobrecarga do cuidador, maior morbidade do idoso e também apresentam correlação com declínio cognitivo mais rápido dos idosos. Além disso, estes sintomas aumentam o risco de institucionalização precoce e também os custos financeiros com assistência médica (BURNS; RABINS, 2000; VEGA et al., 2007; KALAPATAPU; NEUGROSCHL, 2009).

Apesar das dificuldades de desempenho nas atividades cotidianas e do declínio cognitivo do idoso serem pontos de impacto no estresse, desgaste e sobrecarga do cuidador, os aspectos psicológicos e comportamentais são considerados ainda mais impactantes (BURNS; RABINS, 2000; NEIL; BOWIE, 2008; KALAPATAPU; NEUGROSCHL, 2009).

O estresse é definido por Selye (1956 apud NERI 2007) como uma resposta inespecífica a exigências impostas às capacidades físicas e psicológicas de adaptação do individuo. Essas exigências, demandas ou pressões são avaliadas pelo individuo que responderá com maior ou menor intensidade, e de forma que possa senti-lo como agradável (eutress) ou desagradável (distress).

$\mathrm{Na}$ tarefa de cuidar, o cuidador familiar pode sentir prazer e conforto (eutress) quando se sente capaz e gratificado ao desenvolver a atividade, ou pode se sentir tenso, angustiado e sobrecarregado, (distress) quando não atinge seus objetivos ou quando se sente muito pressionado ou a pressão é intolerável (NERI, 2007). É interessante apontar que diante do cuidado diário e das tarefas, o cuidador possa sentir sensações de conforto e de sobrecarga, dependendo de como lida com a situação.

Segundo Guerra (2000) o desgaste é compreendido como alterações orgânicas e psíquicas que um sujeito pode apresentar devido a fatores internos como alterações fisiológicas decorridas da idade e fatores externos.

No presente estudo buscou-se identificar o impacto das alterações psicológicas e comportamentais de idosos com DA, para o desgaste e a sobrecarga dos cuidadores/ familiares, tendo como objetivos específicos traçar o perfil sociodemográfico de um grupo de cuidadores/familiares; verificar a presença, frequência e intensidade das alterações de comportamento nos idosos com DA e o desgaste e a sobrecarga do cuidador diante destas alterações.

\section{MÉTODOS}

Para a realização deste estudo, constitui-se uma amostra de 30 cuidadores/familiares de idosos com DA, de acordo com os critérios do NINCDS-ADRDA (MCKHANN et al., 1984; FROTA et al., 2011). Esses cuidadores foram divididos em grupos de cuidadores de idosos com DA leve $(\mathrm{n}=15)$ e DA moderado $(\mathrm{n}=15)$, de acordo com os critérios do DSM-III-R (APA, 1987). Os critérios do NINCDS$A D R D A$ caracterizam doença de Alzheimer provável com 
base nas seguintes características: início insidioso dos sintomas, alterações cognitivas em memória ou algum outro componente cognitivo, como, linguagem, percepção visuoespacial e funções executivas e a positividade de biomarcadores.

A gravidade da demência foi estabelecida pelos critérios do DSM-III-R (APA, 1987) que considera indivíduos com demência leve aqueles que embora estejam significativamente alterados, mantém capacidade para vida independente e com demência moderada aqueles para os quais a vida independente oferece riscos.

$\mathrm{O}$ estudo foi desenvolvido no SADe (Serviço de Atendimento em Demência) no Departamento de Gestão e Cuidados em Saúde - Campus Baixada Santista da UNIFESP (Universidade Federal de São Paulo) em parceria com a ABRAZ (Associação Brasileira de Alzheimer Regional Santos - SP) e no Ambulatório de Neurologia Cognitiva e do Comportamento da Divisão de Clínica Neurológica do Hospital das Clínicas da Faculdade de Medicina da Universidade de São Paulo (HCFMUSP). No SADe a coleta de dados foi realizada de janeiro a outubro de 2009 e no Ambulatório de Neurologia, no período de março de 2004 a janeiro de 2005.

$\mathrm{O}$ estudo se caracteriza como uma pesquisa exploratória, descritiva e analítica pautada em uma abordagem quantitativa.

Os cuidadores/familiares foram convidados a participar do estudo a partir de um convite feito nos grupos de orientações aos cuidadores em ambos os serviços e os critérios de inclusão foram: ser cuidador/familiar de indivíduos com DA leve e moderada; estar em contato mínimo de 24 horas semanais com o idoso; aceitar participar do estudo assinando o termo de consentimento livre e esclarecido.

Em relação aos aspectos éticos, o projeto, incluindo o termo de consentimento livre e esclarecido, recebeu aprovação do Núcleo de Bioética do Campus Baixada Santista - UNIFESP sob o protocolo - 2091/08 e do Comitê de Ética em Pesquisa do Hospital das Clinicas da Universidade de São Paulo sob o protocolo - 904/03. Os cuidadores/familiares foram esclarecidos acerca dos objetivos do estudo, a partir da leitura do termo de consentimento livre e esclarecido e só foram aplicados os instrumentos após o seu consentimento.

Os cuidadores dos grupos de DA leve e moderada foram pareados para as variáveis: gênero, idade e escolaridade. A porcentagem do pareamento na amostra foi de $73,3 \%$.

Visando a coleta de informações para posterior análise, foram utilizados os seguintes instrumentos:

a) Caracterização sociodemográfica - contém dados de identificação e variáveis sociodemográficas dos cuidadores (nome, idade, gênero, endereço, telefone para contato, escolaridade, grau de parentesco com o indivíduo com DA);

b) Inventário Neuropsiquiátrico (INP) (CUMMINGS et al., 1994; CAMOZZATO et al., 2008) - têm por objetivo obter informações sobre a presença, frequência e intensidade das alterações psicológicas e comportamentais. As informações são colhidas com os cuidadores;

c) Inventário Neuropsiquiátrico - escala de desgaste do cuidador (INP-D) (CUMMINGS et al., 1994; CAMOZZATO et al., 2008) - mede o desgaste dos cuidadores diante de cada domínio dos sintomas avaliados pelo INP.

d) Inventário de sobrecarga do cuidador - avalia a sobrecarga do cuidador considerando as seguintes áreas: saúde do cuidador, bem-estar psicológico, finanças, vida social e relacionamento entre o cuidador e o paciente (ZARIT et al., 1987; TAUB et al., 2004).

A coleta de dados com cada cuidador foi realizada por meio de entrevista em apenas 01 encontro com 1 hora e 30 minutos de duração, em média.

Foram utilizados os programas EXCEL 2000 e o SPSS 10.0 (Statistical Package for the Social Sciences) para a confecção do banco de dados e realização das análises descritivas. Para a análise da correlação entre os SPCD, desgaste e a sobrecarga foram utilizados os coeficientes de correlação de Pearson. Foi considerado como nível de significância um valor de $p<0,05$.

\section{RESULTADOS}

A média de idade dos idosos com DA na fase leve foi de $81 \pm 5,5$ anos e a média de escolaridade foi de $9 \pm$ 5,2 anos.

No grupo de DA moderada, a média de idade foi de $80 \pm 6,7$ anos e a média de escolaridade foi de $5 \pm 5$ anos.

Há um predomínio de idosos do gênero feminino em ambos os grupos, $66,6 \%$ no grupo de DA leve e $53,3 \%$ no grupo de DA moderada.

Em relação aos cuidadores também há uma predominância do gênero feminino em ambos os grupos, $93,3 \%$ no grupo de cuidadores de idosos com DA leve e $73,3 \%$ no grupo de cuidadores de DA moderada.

A média de idade dos cuidadores foi de $64,3 \pm 12,6$ anos no grupo de DA leve e $60,3 \pm 15$ anos no grupo de DA moderada.

A média de escolaridade dos cuidadores foi de 11 $\pm 3,5$ anos (DA leve) e 10,2 $\pm 3,6$ anos (DA moderada).

Os Gráficos 1 e 2 mostram a prevalência dos SPCD na amostra total e por grupos de gravidade. 


\section{Gráfico 1 - Prevalência dos SCPD na amostra total (\%)}

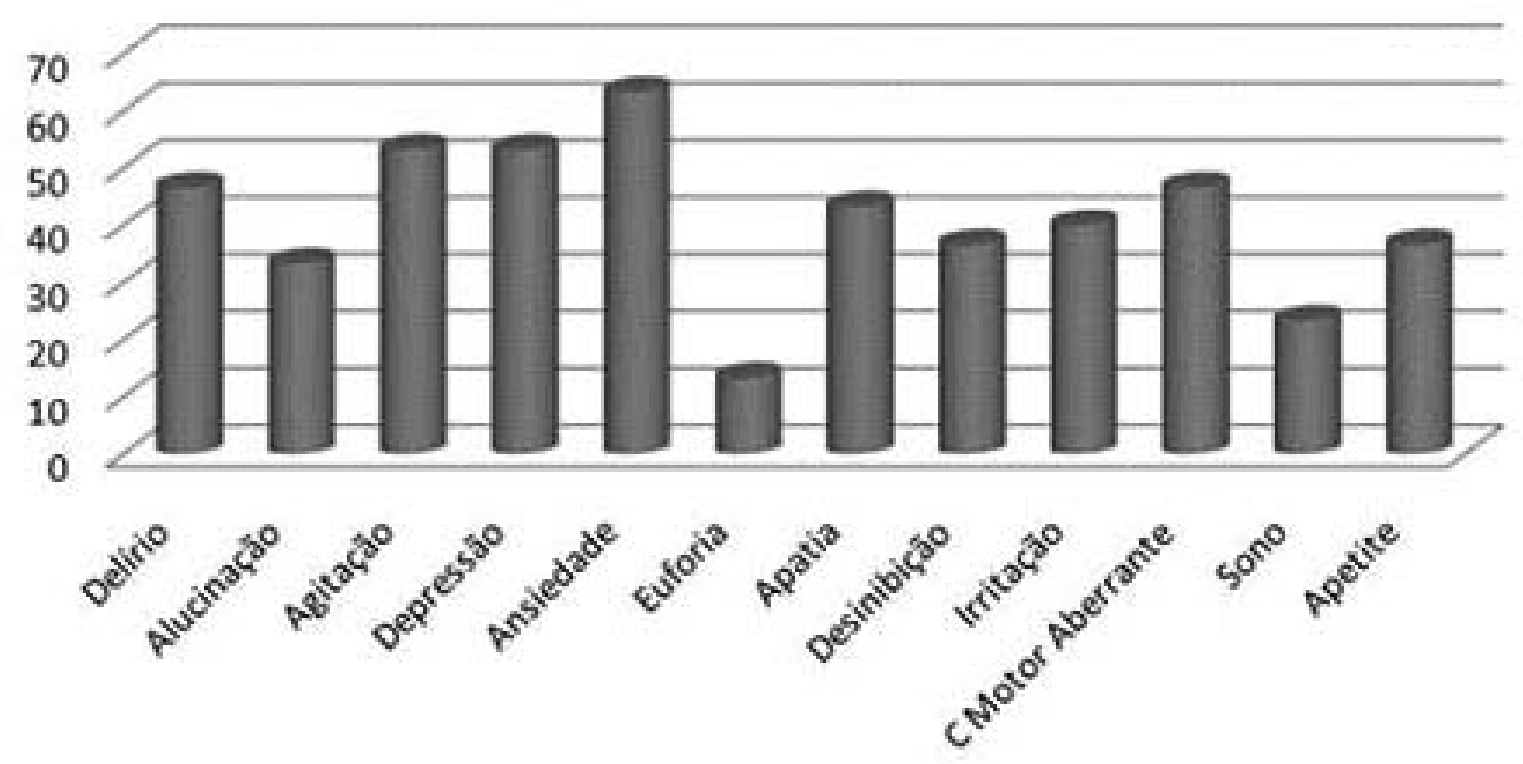

Prevalência Total (96)

Gráfico 2 - Prevalência dos SCPD na fase leve e moderada (\%)

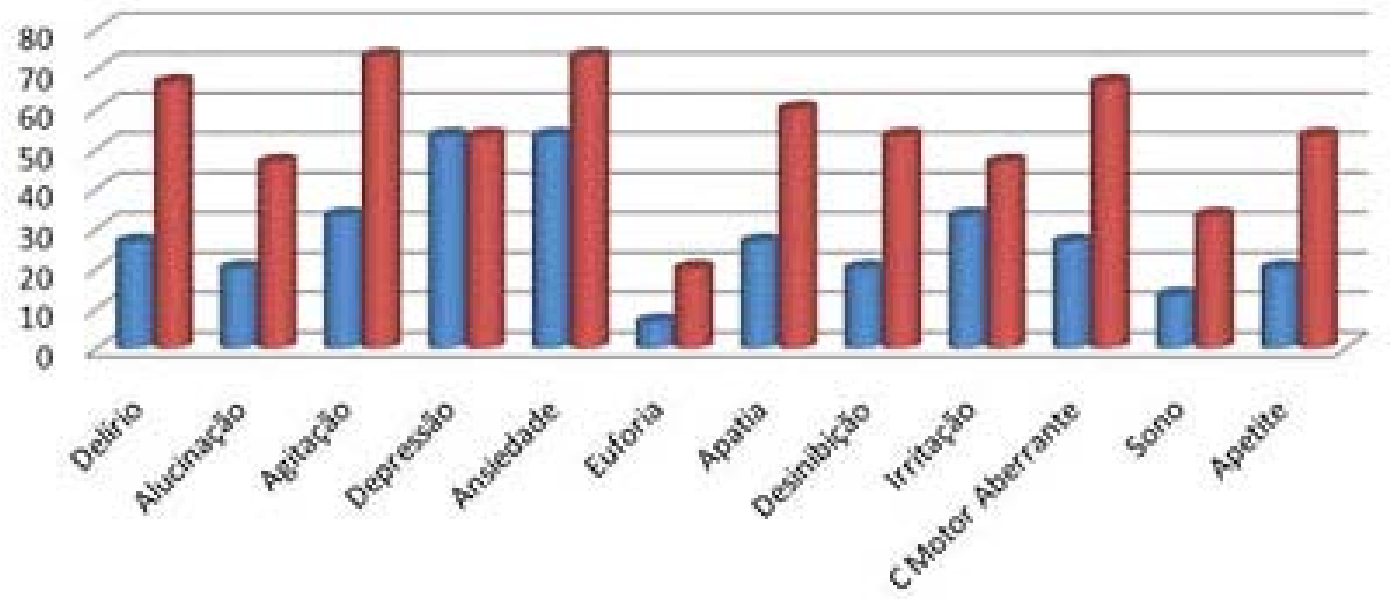

= Prevalência na fase leve (\%) $\quad$ : Prevalência na fase moderada (\%) 
O sintoma mais prevalente, na amostra total, foi ansiedade (63\%), seguido de agitação e depressão (53\%), delírio e comportamento motor aberrante (46\%) e apatia $(43 \%)$.

$\mathrm{Na}$ fase leve, os comportamentos mais prevalentes foram depressão e ansiedade (53,3\%), seguido de agitação e irritação $(33,3 \%)$ e o comportamento com menor frequência foi o de euforia $(6,7 \%)$.

$\mathrm{Na}$ fase moderada, os comportamentos mais prevalentes foram agitação e ansiedade $(73,3 \%)$, seguido de delírio e comportamento motor aberrante $(66,7 \%)$, apatia $(60 \%)$ e o comportamento com menor prevalência foi o de euforia $(20 \%)$.

Na Tabela 1 são apresentadas as médias de pontuações obtidas por meio da aplicação do INP, INP-D e inventário de sobrecarga aos cuidadores de idosos com DA leve e moderada.

Quando comparadas, as médias de pontuação do INP e do INP-D nas fases leve e moderada da doença, nota-se que as médias de pontuação são maiores na fase moderada.

A Tabela 2 apresenta os coeficientes de correlação entre os sintomas psicológicos e comportamentais listados no INP, o desgaste do cuidador e a sobrecarga do cuidador/familiar nas fases leve e moderada da doença.

Tabela 1 - Médias e desvio-padrão das pontuações no INP-D, INP e Inventário de sobrecarga

\begin{tabular}{lcc}
\hline Variáveis & $\begin{array}{c}\text { Cuidadores/ } \\
\text { Familiares } \\
\text { (DA leve) }\end{array}$ & $\begin{array}{c}\text { Cuidadores/Familiares } \\
\text { (DA moderada) }\end{array}$ \\
\hline INP-D & $8,5^{\mathrm{a}} \pm 8,8^{\mathrm{b}}$ & $22,4^{\mathrm{a}} \pm 13,3^{\mathrm{b}}$ \\
INP & $15,7^{\mathrm{a}} \pm 19,0^{\mathrm{b}}$ & $51,0^{\mathrm{a}} \pm 32,0^{\mathrm{b}}$ \\
Inventário de & $29,0^{\mathrm{a}} \pm 11,5^{\mathrm{b}}$ & $44,0^{\mathrm{a}} \pm 14,0^{\mathrm{b}}$ \\
sobrecarga & & \\
\hline
\end{tabular}

${ }^{\mathrm{a}}$ Médias ${ }^{\mathrm{b}}$ Desvios-padrão

Tabela 2 - Correlação entre desgaste e a sobrecarga dos cuidadores e os sintomas psicológicos e comportamentais dos idosos

\begin{tabular}{llllc}
\hline \multirow{2}{*}{ SCPD } & \multicolumn{2}{c}{ Desgaste } & \multicolumn{2}{c}{$\begin{array}{c}\text { Sobrecarga } \\
\text { Movederada }\end{array}$} \\
\hline Delírio & Moderada & $0,74^{* *}$ & $0,65^{* *}$ & 0,25 \\
Alucinação & $0,57^{* *}$ & $0,80^{* *}$ & 0,20 & 0,40 \\
Agitação & 0,49 & 0,46 & 0,44 & 0,06 \\
Depressão & $0,77^{* *}$ & 0,14 & 0,09 & $-0,08$ \\
Ansiedade & $0,68^{* *}$ & 0,14 & 0,31 & $-0,16$ \\
Euforia & $0,65^{* *}$ & $0,56^{*}$ & 0,31 & 0,40 \\
Apatia & 0,43 & 0,51 & $-0,19$ & $0,65^{* *}$ \\
Desinibição & $-0,04$ & $0,79^{* *}$ & 0,27 & $-0,01$ \\
Irritação & $0,70^{* *}$ & 0,10 & 0,26 & 0,24 \\
Comportamento Motor & $0,68^{* *}$ & $0,58^{*}$ & 0,40 & 0,41 \\
Aberrante & 0,19 & $0,56^{*}$ & $-0,01$ & 0,35 \\
Sono & 0,18 & $0,58^{*}$ & 0,23 & 0,29 \\
Apetite total & 0,22 & $0,58^{*}$ & & \\
\hline
\end{tabular}

${ }^{*} p<0,05{ }^{* *} p<0,01$

Pode-se observar que para os indivíduos na fase leve da doença, os comportamentos que se correlacionaram com o desgaste do cuidador/familiar foram agitação, seguido de desinibição, irritação e depressão.

$\mathrm{Na}$ fase moderada da doença, o comportamento que se mais se correlacionou com o desgaste foi alucinação, seguido de apatia e delírio.

Em relação aos comportamentos que mais sobrecarregaram, na fase leve foi o delírio e na fase moderada a apatia.

Na análise de associação entre os sintomas psicológicos e comportamentais na demência e o desgaste dos cuidadores, foram identificadas correlações significativas em ambos os grupos ( $r=0,90$ (DA leve) e $r=0,77$ (DA moderada), com $p<0,01)$.

$\mathrm{Na}$ análise de associação entre os sintomas psicológicos e comportamentais na demência e a sobrecarga dos cuidadores foi possível identificar correlações significativas em ambos os grupos ( $r=0,55$ (DA leve) e $r=0,59$ (DA moderada, com $\mathrm{p}<0,01)$ ).

\section{DISCUSSÃO}

Em relação às características sociodemográficas da amostra estudada, pode-se observar que a média da idade dos idosos com DA, tanto na fase leve como na moderada foi de 
aproximadamente 80 anos e de seus cuidadores/familiares foi de aproximadamente 62 anos. Isto pode apontar para um cuidador em processo de envelhecimento, com suas próprias peculiaridades, o que vem a ser mais um aspecto a ser considerado na presença de desgaste e sobrecarga, pois além de se dedicar ao cuidado do idoso com DA terá que cuidar de si próprio.

A maioria dos cuidadores familiares entrevistados é do gênero feminino, sendo que a maior porcentagem foi de esposas e filhas. Este resultado está de acordo com as evidências de diversos estudos anteriores (INOUYE et al., 2009; NOVELLI et al., 2010).

Segundo Cruz e Hamdan (2008), os cônjuges são os que sofrem o maior impacto do cuidado. Uma explicação para isto é que, devido à sua idade mais avançada, o cônjuge está mais suscetível a problemas de saúde, fator relacionado a um maior impacto. Cuidadores mulheres costumam sofrer um impacto maior, pois assumem com mais frequência tarefas desgastantes, como a higiene do idoso, além de terem que gerenciar as tarefas domésticas.

Quando comparados os dados sociodemográficos dos cuidadores nos dois grupos, os resultados não mostram grandes diferenças entre idade, gênero, escolaridade e grau de parentesco. Isto vem demonstrar que a amostra utilizada nesta pesquisa é homogênea. Cabe mencionar que os cuidadores desta amostra apresentam uma média de escolaridade alta, de aproximadamente $\pm 11,0$ anos.

$\mathrm{Na}$ amostra total, a alteração mais prevalente foi ansiedade (63\%), seguido de agitação e depressão (53\%), delírio e comportamento motor aberrante $(46 \%)$ e apatia (43\%). As prevalências encontradas pelo estudo se aproximam de outros estudos executados: ansiedade (VEGA et al., 2007), depressão (TATSCH et al., 2006; VEGA et al., 2007; SHIMABUKURO et al., 2008), delírio (VEGA et al., 2007), comportamento motor aberrante (SHIMABUKURO et al., 2008), apatia (LYKETSOS et al., 2002; TATSCH et al., 2006, CARAMELLI; BOTTINO, 2007).

Comparando-se a prevalência nos dois grupos, observa-se que os SPCD acentuaram-se nos indivíduos na fase moderada da doença. Apenas o comportamento de depressão apresentou a mesma prevalência nos dois grupos.

Lyketsos et al. (2000) comparou a prevalência dos sintomas de acordo com a fase da doença e encontrou que somente a agitação e comportamento motor aberrante eram mais comuns nos idosos em fases posteriores. No entanto, vários outros distúrbios (delírios, ansiedade, apatia, irritabilidade, euforia e desinibição) foram relatados com gravidade semelhante em todas as fases da demência.

Hamuro et al. (2007) verificou a prevalência dos sintomas ao longo dos três estágios da doença e encontrou que a depressão, agitação e comportamento motor aberrante foram estatisticamente associados com a gravidade da DA. A prevalência dos Sintomas Psicológicos e Comportamentais na Demência (SPCD) como um amplo grupo de sintomas não aumentou com o aumento da severidade da doença.

A diferença nos resultados pode ser explicada porque o presente estudo avaliou os SPCD pontualmente nas fases leve e moderada da DA, diferentemente desses estudos que verificaram a progressão nas três fases da doença.

Shimabukuro et al. (2008) encontraram que as frequências de euforia e comportamento motor aberrante foram significativamente relacionadas com o nível de gravidade da DA e observou também que os pacientes com DA em fase leve tinham delírios, alucinações, agitação, depressão, ansiedade, apatia e irritabilidade tão frequentemente quanto aqueles na fase moderada ou grave.

Senanarong et al. (2004) encontraram que quanto mais grave a DA, maior a prevalência de agitação. De acordo com o estudo de Fuh et al. (2005), a prevalência de delírios, alucinações e comportamento motor aberrante aumentaram significativamente com a gravidade da demência em pacientes com DA.

Nota-se que a prevalência dos SPCD pode variar bastante, dependendo do tipo de amostra estudada e dos métodos utilizados para avaliá-los (CARAMELLI; BOTTINO, 2007), o que explica as diferentes prevalências e associações encontradas pelos estudos realizados.

Verifica-se que existem correlações significativamente estatísticas entre a presença e intensidade dos sintomas com o desgaste do cuidador, sendo que a correlação foi maior na fase leve da doença. A correlação encontrada pode ser explicada pelo fato de que na fase leve da doença, o cuidador passa a entrar em contato com as dificuldades advindas com a doença e consequentemente com os SPCD. Esse contato pode ocasionar desgaste no cuidador, por não entender e saber lidar com esses comportamentos.

Ao se comparar as médias de pontuações dos sintomas avaliados na fase leve e moderada, observa-se que há uma diferença entre elas. Na fase leve da doença, a média se mostra bem inferior que na fase moderada. O resultado está de acordo com o estudo de Shimabukuro et al. 2008, em que a gravidade dos SPCD aumenta com o aumento da severidade da demência.

Os comportamentos que mais desgastaram o cuidador diferenciaram-se de acordo com a fase da doença.

$\mathrm{Na}$ fase leve os sintomas que mais se associaram com o desgaste foram agitação, seguido de desinibição, irritação e depressão. Na fase moderada da doença, os sintomas que se mais se associaram foram alucinação, seguido de apatia e delírio.

Analisando-se os resultados obtidos com a associação entre o desgaste e o SPCD e com a frequência dessas 
alterações, observa-se que os sintomas que apresentaram a maior prevalência não foram os que mais desgastaram.

Os SPCD da amostra total que mais desgastaram o cuidador foram à ansiedade e a agitação $(36 \%)$, seguido de comportamento motor aberrante $(30 \%)$.

Nossos resultados vão ao encontro a outros estudos, como o de Camozzato et al., (2008) em que encontrou a apatia (32\%) como a alteração que causou acentuado e extremo desgaste, seguido de agitação (25\%), delírio e ansiedade $(22 \%)$. Tatsch et al. (2006) encontrou que as causas de desgaste do cuidador foram delirio, agitação, comportamento motor aberrante e desinibição. De acordo com o estudo de Godinho et al. (2008), a apatia causou o maior nível de desgaste, seguida de agitação e agressividade.

Pode-se observar que o desgaste foi maior com a progressão da doença em quase todos os sintomas, exceto a euforia que foi mais desgastante na fase leve da DA.

Apesar do delírio e apatia não serem os comportamentos mais prevalentes, neste estudo, são as alterações que mais se associaram a sobrecarga do cuidador.

Segundo Engelhardt et al. (2005), o cuidador desempenha um papel essencial na vida diária dos idosos com DA, envolvendo-se em praticamente todos os aspectos do cuidado e assumindo responsabilidades adicionais de maneira crescente. A medida que a doença progride, maior é o número de tarefas que este cuidador terá que assumir, aumentando assim, sua sobrecarga.

Pelos resultados do estudo podemos identificar que nem todos os sintomas se associaram com a sobrecarga de cuidado e, além disso, a sobrecarga do cuidador pode incluir fatores mais amplos do que apenas o desgaste em relação aos SPCD e a tarefas recentes relativas ao cuidado. A sobrecarga pode estar relacionada também a outras tarefas cotidianas, papéis ocupacionais e demandas que se sobrepõe e a outras variáveis que compõem a vida do individuo além do cuidado.

Como limitação do estudo, temos que o tamanho da amostra é pequeno, os resultados obtidos se aplicam apenas a essa amostra, não permitindo a sua generalização.

Os SPCD se associam com maiores taxas de institucionalização, maior sobrecarga dos cuidadores e menor sobrevida de pacientes com DA. Portanto, para que esta situação seja amenizada é importante que sejam pensadas estratégias de intervenção farmacológicas e não farmacológicas que auxiliem estes cuidadores (CARAMELLI; BOTTINO, 2007) no manejo diário destes sintomas em idosos.

O conhecimento da amplitude de tarefas que cerca o papel do cuidador e suas repercussões sobre a vida do mesmo são de vital importância para o desenvolvimento de políticas públicas tanto para a abordagem dos sintomas psicológicos e comportamentais dos idosos, incluindo a proposição de abordagens farmacológicas e não farmacológicas, bem como estratégias de minimização do desgaste e da sobrecarga dos cuidadores, considerando a integralidade do cuidado na díade - idoso e cuidador/familiar.

\section{CONCLUSÕES}

Em relação ao perfil sociodemográfico, a maioria dos cuidadores é do gênero feminino com a média de idade superior a 60 anos, sendo que as mulheres e filhas compreendem a maioria dos cuidadores.

A análise da presença dos Sintomas Psicológicos e Comportamentais (SPCD), na amostra total, mostrou que os sintomas mais prevalentes foram ansiedade, agitação, depressão e delírio.

$\mathrm{Na}$ análise por grupos, na fase leve, os comportamentos mais prevalentes foram depressão e ansiedade. Em relação ao desgaste do cuidador/familiar, os sintomas que mais se associaram foram seguidos de desinibição, irritação e depressão. O delírio se associou tanto com o desgaste como com a sobrecarga dos cuidadores.

$\mathrm{Na}$ fase moderada, os comportamentos mais prevalentes foram ansiedade e agitação, seguida de delírio. Em relação ao desgaste do cuidador os sintomas que mais se associaram foram à alucinação, seguido de apatia e delírio. A apatia também se associou a sobrecarga do cuidador.

Devido ao desgaste e sobrecarga de cuidado que os cuidadores de idosos com DA apresentam, tanto em relação aos sintomas psicológicos e comportamentais, como também devido aos outros fatores que advém com a doença, faz-se necessário e urgente, a implementação de politicas publicas que visem o atendimento e acompanhamento do idoso e de seus cuidadores/familiares.

ARAKAKI, B. K.; TSUBAKI, J. N. S.; CARAMELLI, P.; NITRINI, R.; NOVELLI, M. M. P. C. Analysis of caregiver burden of elderly with Alzheimer disease caused by behavioral psychological symptons of dementia. Rev. Ter. Ocup. Univ. São Paulo, v. 23, n. 2, p. 113-121, maio/ago. 2012.

\begin{abstract}
Alzheimer's disease (AD) is characterized as a degenerative disease that affects many domains, including behavior and can cause burden in caregivers/family member of these individuals. The aim of the study was to analyze the caregiver burden in relation to the presence and intensity of Behavioral and Psychological Symptoms in Dementia (BPSD). We used socio demographic
\end{abstract}


profile, Neuropsychiatric Inventory (NPI) and NPI - caregiver distress (NPI-D) for our evaluation. We interviewed 30 caregivers of elderly with Alzheimer disease according to NINCDS-ADRDA criteria, divided in mild level $(n=15)$ and moderate level $(n=15)$ according to DSM-III-R criteria. The most prevalent symptoms were, in the mild level, anxiety and depression, and in the moderate level they were anxiety and agitation. The analyses conducted between caregivers' distress and BPSD showed us that the symptoms with the greatest correlation in the mild level were agitation followed by disinhibition. In relation to caregiver burden there was a significant correlation with delirium in mild level and apathy in moderate level. In conclusion, it was observed that the BPSD that affect the burden caregivers differ according to the level of the disease and the knowledge of these impacts allows us to reflect and propose actions to implement public policies and interventions in the care of the elderly and their caregivers/family members.

KEYWORDS: Alzheimer disease; Caregivers/psychology; Caregivers/utilization; Family; Aged; Behavioral symptoms/psychological; Behavioral symptoms/diagnosis.

\section{REFERÊNCIAS}

\section{AMERICAN PSYCHATRIC DIAGNOSTIC AND STATISTICAL MANUAL OF MENTAL DISORDERS. 3rd ed. Washington, DC: American Psychiatric Association, 1987.}

ANGHINAH, R.; et al. Alterações psíquicas e comportamentais em demência. In: FORLENZA, O. V. Psiquiatria geriátrica. São Paulo: Atheneu, 2007. p. 207-214.

BURNS, A.; RABINS, P. Carer burden in dementia. Int. J. Geriat. Psychiatry, v. 15, p. 9-13, 2000.

CAMARANO, A. A. Envelhecimento da população brasileira: uma contribuição demográfica. In: FREITAS, E. V.; Py, L, ed. Tratado de geriatria e gerontologia. Rio de Janeiro: Guanabara Koogan, 2006. p. 88-104.

CAMOZZATO, A. L.; et al. Reliability of the brazilian portuguese version of the neuropsychiatric inventory (NPI) for patients with Alzheimer's disease and their caregivers. Int. Psychogeriat., v. 20, p. 383-393, 2008.

CARAMELLI, P.; BARBOSA, M. T. Como diagnosticar as quatro causas mais frequentes de demência? Rev. Bras. Psiquiatr., v. 24, n. 1, p.7-10, 2002.

CARAMELli, P.; BOTTINO, C. M. C. Tratando os Sintomas comportamentais e psicológicos da demência (SCPD). J. Bras Psiquiatr., v. 56, p. 83-87, 2007.

CUMMINGS, J. L.; MEGA, M.; GARY, K. The neuropsychiatric inventory: comprehensive assessment of psychopathology in dementia. Neurology, v. 44, p. 2308-2314, 1994.

CRUZ, M. N.; HAMDAN, A. C. O impacto da doença de Alzheimer no cuidador. Psicol. estud., v. 13, p. 223-229, 2008.

ENGELHARDT, E.; DOURADO, M.; LACKS, J. A doença de Alzheimer e o impacto nos cuidadores. Rev. Bras. Neurol., v. 14, n. 2, p. 5-11, 2005.

FREITAS, I. C. C.; et al. Convivendo com o portador de Alzheimer: perspectivas do familiar cuidador. Rev. Bras. Enferm.; v. 61, n. 4, p. 508-513, 2008.

FROTA, N. A. F.; et al. Criteria for the diagnosis of Alzheimer's disease. Dement. Neuropsychol.; v. 5, n. 3, p. 146-152, 2011.

FUH, J. L.; WANG. S. J.; CUMMINGS, J. L. Neuropsychiatric profiles in patients with Alzheimer's disease and vascular dementia. J. Neurol. Neurosurg. Psychiatry, v. 76, p. 1337-1341, 2005.

GARRIDO, R.; MENEZES, P. R. Impacto em cuidadores de idosos com demência atendidos em um serviço psicogeriátrico. Rev Saúde Pública, v. 38, n. 6, p. 835-841, 2004.

GUERRA, C. I. C. O. Fatores de risco para desgaste do cuidador familiar de paciente na fila do transplante cardíaco. 2000. Dissertação (Mestrado). Escola de Enfermagem, Universidade de São Paulo, São Paulo, 2000.

GODINHO, C.; et al. Association of caregiver demographic variables with neuropsychiatric symptoms in Alzheimer's disease patients for distress on the Neuropsychiatric Inventory (NPI). Dement. Neuropsychol., v. 2, n. 3, p. 211-216, 2008.

HAMURO, A.; et al. Behavioral and psychological symptoms of dementia in untreated Alzheimer's disease patients. Psychogeriatrics, v. 7, p. 4-7, 2007.

HERRERA, E. Jr.; et al. Epidemiological survey of dementia in community-dwelling Brazilian population. Alzheimer Dis. Assoc. Disord., v. 16, p. 103-108, 2002.

INOUYE, K.; Pedrazzani ES, Pavarini SC; Toyoda, C. Y. Perceived quality of life of elderly patients with dementia and family caregivers: evaluation and correlation. Rev. Latino-Am. Enfermagem, Ribeirão Preto, v. 17, n. 2, p. 187-193, 2010.

KALAPATAPU, R. K.; NEUGROSCHL, J. A. Update on neuropsychiatric symptoms of dementia: Evaluation and management. Geriatrics, v. 64, n. 4, p.20-26, 2009.

LYKETSOS, C. G.; et al. Mental and behavioral disturbances in dementia: findings from the cache country study on memory in 
aging. Am. J. Psychiatry, v. 157, n. 5, p. 708-714, 2000.

LYKETSOS, C. G.; et al. Prevalence of Neuropsychiatric Symptoms in Dementia and Mild Cognitive Impairment: results from the cardiovascular health study. JAMA, v. 288, p. 1475-1483, 2002.

MCKCKANN, G.; et al. Clinical diagnosis of Alzheimer's disease: report of the NINCDS-ADRDA work group under the auspice of Department of Health and Human Services Task Force on Alzheimer's disease. Neurology, v. 34, p. 939-944, 1984.

NEIL, W.; BOWIE, P. Carer burden in dementia - assessing the impact of behavioural and psychological symptoms via self-report questionnaire. Int. J. Geriatr. Psychiatry, v. 23, p. 60-64, 2008.

NERI, A. L. Bem-estar e estresse em familiares que cuidam de idosos fragilizados e de alta dependência. In: NERI, A. L. Qualidade de vida e idade madura. Campinas: Papirus Editora, 2007. p. 237-285.

NOVELLI, M. M. P. C.; NITRINI, R.; CARAMELLI, P. Cuidadores de idosos com demência: perfil sociodemográfico e impacto diário. Rev. Ter. Ocup. Univ. São Paulo, v. 21, n. 2, p. 139-147, 2010.

SHIMABUKURO, J.; AWATA, S.; MATSUOKA, H. Behavioral and psychological symptoms of dementia characteristic of mild Alzheimer patients. Seishin Shinkeigaku Zasshi, v. 110, n. 7, p. 529-535, 2008.

TATSCH, M. F.; et al. Neuropsychiatric symptoms in Alzheimer disease and cognitively impaired, nondemented elderly from a community-based sample in Brazil: prevalence and relationship with dementia severity. Am. J. Geriatr. Psychiatry, v. 14, n. 5, p. 438-445, 2006.

TAUB, A.; BERTOLUCCI, P. H.; ANDREOLI, S. B. Dementia caregiver burden: reliability of the Brazilian version of the Zarit caregiver burden interview. Cad. Saúde Pública, v. 20, p. 372376, 2004.

VEGA, U. M.; MARINHO, V.; ENGELHARDT, E.; LAKS, J. Sintomas neuropsiquiátricos nas demências: Relato preliminar de uma avaliação prospectiva em um ambulatório do Brasil. Arq Neuropsiquiatr., v. 65, n. 2-B, p. 498-502, 2007. 